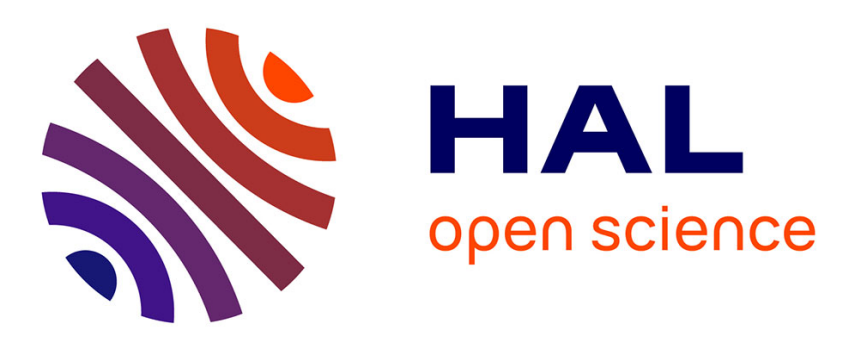

\title{
Constitutive relation error estimators for (visco) plastic finite element analysis with softening
}

Pierre Ladevèze, Nicolas Moës, B. Douchin

\section{To cite this version:}

Pierre Ladevèze, Nicolas Moës, B. Douchin. Constitutive relation error estimators for (visco) plastic finite element analysis with softening. Computer Methods in Applied Mechanics and Engineering, 1999, 176, pp.247-264. 10.1016/S0045-7825(98)00340-5 . hal-02109619

\section{HAL Id: hal-02109619 https://hal.science/hal-02109619}

Submitted on 25 Apr 2019

HAL is a multi-disciplinary open access archive for the deposit and dissemination of scientific research documents, whether they are published or not. The documents may come from teaching and research institutions in France or abroad, or from public or private research centers.
L'archive ouverte pluridisciplinaire HAL, est destinée au dépôt et à la diffusion de documents scientifiques de niveau recherche, publiés ou non, émanant des établissements d'enseignement et de recherche français ou étrangers, des laboratoires publics ou privés. 


\title{
Constitutive relation error estimators for (visco)plastic finite element analysis with softening
}

\author{
Pierre Ladevèze ${ }^{\mathrm{a}, *}$, Nicolas Moës ${ }^{\mathrm{b}}$, Bernard Douchin ${ }^{2}$ \\ a Laboratoire de Mécanique et Technologie, E.N.S. Cachan/C.N.R.S./Université Paris 6, 61, Avenue du Président Wilson, \\ 94235 Cachan Cedex, France \\ ${ }^{\mathrm{b}}$ The Texas Institute for Computational and Applied Mathematics, The University of Texas at Austin, Austin, TX 78712, USA
}

\begin{abstract}
A posteriori error estimators based on constitutive relation residuals have been developed for 20 years, in particular at Cachan. This approach has a strong physical meaning and is quite general. Here, we introduce an extended constitutive relation error estimators family able to measure the quality of finite element computations of structures which exhibit plastic/viscoplastic behavior with softening. These measures take into account, over the studied time interval, all the classical error sources involved in the computation : the space discretization (the mesh), the time discretization and the iterative technique used to solve the nonlinear discrete problem.
\end{abstract}

\section{Introduction}

Nowadays, numerical simulations are used to solve more and more complex problems. The control of such computations has become a critical issue for their performance. Concerning a posteriori error estimate for the finite element approximation of linear elliptic problems, a vast literature is available. Three main approaches must be distinguished. Chronologically speaking, the first one is based on the concept of error in the constitutive law [1]. This concept is a priori independent of the type of numerical approximation used. Its application in the case of finite element approximations may be found in [1-3]. The second approach, introduced by Babuska and Rheinboldt [4,5], then developed by Zienkiewicz et al. [6,7] and more recently by Oden et al. [8,9], uses the equilibrium residuals through local problems to estimate the error. The last one, developed by Zienkiewicz and Zhu [10-12], consists of comparing the finite element solution to a smoother one obtained by special averaging techniques. A validation of these a posteriori error estimators may be found in [13,14].

Here, we are interested in plastic and viscoplastic problems with softening under the small strains, small displacements and isothermal assumptions. These problems are time-dependent nonlinear problems. The literature available is much narrower compared to the linear case. Also, it is important to distinguish between the nonlinear time-dependent and time-independent problems. Concerning the later type, let us mention Babuska [15] for the design of estimates for nonlinear elasticity problems of rods, Johnson [16] for Hencky-type plasticity problems, and Verfurth for the proof of bounds on the error [17]. For nonlinear time-dependent problems, viscoplasticity problems were treated in [18], strain localization problems in [19-21] and large strains problems in [22-25]. In most cases, techniques devised for linear problems or time-independent nonlinear problems are used at each time step so that the error due to the time discretization may hardly be taken into account. Indeed, in nonlinear time-dependent problems, three error sources must be distinguished: the space discretization, the time discretization and the iterative technique used to solve the nonlinear discrete problem.

Two major error estimators based on the constitutive law residuals have been introduced in Cachan for nonlinear time-dependent problems: the Drucker error and the dissipation error. They have been applied to

*Corresponding author. 
plastic and viscoplastic problems and have both in common the fact that they are able to take into account the three error sources mentioned above. The Drucker error is based on a sufficient condition insuring the stability of the material (Drucker's inequality [26]). This error was introduced in [27], first applied to plane stress problems [28] and used in adaptive procedure in [29,30]. The dissipation error takes into account the more and more common situation in which the material behavior is described by internal variables. It has been elaborated in [31,32], first applied in [33], and used in adaptive procedure in [30,34]. It was named dissipation error since the error is estimated through the residuals of the laws describing the evolution of the state of the material, thus the dissipation process. In this approach the state laws are a priori satisfied allowing an extension of the Prager-Synge theorem [32].

The Drucker error assumes that the functional law linking the present stress state to the strain history satisfies Drucker's stability condition. This assumption is true in general for stable visco-plastic behavior but it is not true for softening material. On the other hand, by enforcing a priori the verification of the state laws, the dissipation error is not flexible enough to handle material with softening. This is why we introduce a new constitutive relation error estimators family able to take into account a general (visco)plastic behavior with softening. This new family will have the viscoplastic case with no-softening, the elastic case with softening and the pure elastic case as particular cases. The idea is to measure the error not only on the evolution laws but also on the state laws. Thus, the state laws do not need to be satisfied a priori, introducing more flexibility.

The plan of the paper is as follows. In Section 2, the reference problem and the formulation of the material behavior for (visco)plastic material with softening is detailed. Section 3 introduces the new family of constitutive residual error estimators for general (visco)plastic behavior with softening. Then, in Section 4, the implementation of the errors in the finite element framework is explained. numerical experiments are carried out in Section 5; first without, then with softening. The influence of the numerical parameters (mesh, time step and stopping criteria) on the value of the errors are also analyzed in this section.

\section{The model problem}

Concerning the notations, vectors are underlined $(u, \underline{v}, \ldots)$ and second-order tensors are bold Greek. For instance, the stress and strain tensors are denoted by $\boldsymbol{\sigma}$ and $\boldsymbol{\epsilon}$, respectively. This notation is also used to denote the additional internal variables. More complex operators are in capital bold (e.g. $\boldsymbol{K}$ for the Hooke's tensor). $\mathrm{Tr}$ stand for the trace operator and $t$ in exponent for the usual transposition. The dot ' ' denotes the scalar product between vectors and the colon ' $:$ ' the dot product of second-order tensors. $\nabla \underline{v}$ defines the gradient of the vector $\underline{v}$. In an orthonormal basis, we have $[\nabla \underline{v}]_{i j}=v_{i, j}$. Finally, the first derivative of a function $f$ with scalar argument is denoted by $f^{\prime}$.

The solid medium under study occupies a domain $\Omega$, bounded by $\partial \Omega$, which is independent of the time $t$ (small strains and displacements assumption). The environment of the medium is schematized for all $t \in[0, T]$, with an imposed displacement $\underline{u}_{d}$ on a part $\partial_{1} \Omega$ of the boundary, a traction $\underline{F}_{\mathrm{d}}$ on $\partial_{2} \Omega$ (complementary to $\partial_{1} \Omega$ ), and a body load $f_{\mathrm{d}}$ on the domain $\Omega$. The partition of $\partial \Omega$ in $\partial_{1} \Omega$ and $\partial_{2} \Omega$ is assumed constant in time. $U$ denotes the space of the displacement field $\underline{u}$ defined on $\Omega$, $\mathscr{E}$ the space of strain field $\epsilon$ defined on $\Omega$ and $\mathscr{S}$ the space of the stress field $\sigma$, also defined on $\Omega$. The extensions of these three spaces to the entire time range $[0, T]$ will be denoted $\mathcal{U}^{[0, T]}, \mathscr{E}^{[0, T]}$ and $\mathscr{S}^{[0 . T]}$, respectively.

The model problem involves the kinematic constraints, the equilibrium equations and the constitutive equations describing the behavior of the material.

\subsection{Kinematic constraints and equilibrium equations}

Under the small displacement and strain assumptions, the kinematic and static constraints are:

- the displacement solution, $\underline{u}$, must belong to the space $\mathcal{U}_{a, t]}^{[\bullet, T]}$ :

$$
\underline{u} \in \mathcal{U}_{a d}^{[0 . T]}=\left\{\underline{v} \in \mathcal{U}^{[0, T]}: \underline{v}=\underline{u}_{\mathrm{d}} \text { on } \partial_{1} \Omega \times[0, T]\right\}
$$


- the strain solution, $\epsilon$, must belong to space $\mathscr{E}_{a d}^{[0 . T]}$ :

$$
\epsilon \in \mathscr{E}_{a d}^{[0, T]}=\left\{\gamma \in \mathscr{E}^{(0, T)}: \exists \underline{v} \in \mathscr{U}_{a d}^{[0, T]}: \gamma=\frac{1}{2}\left(\nabla \underline{v}+(\nabla \underline{v})^{t}\right)\right\}
$$

- the stress solution, $\boldsymbol{\sigma}$, must belong to space $\mathscr{S}_{a d}^{[0 . T]}$ :

$$
\boldsymbol{\sigma} \in \mathscr{S}_{a d}^{[0, T]}=\left\{\tau \in \mathscr{P}^{[0, T]}: \int_{\Omega} \tau: \epsilon(\underline{v}) \mathrm{d} \Omega-\int_{\Omega} f_{\mathrm{d}} \cdot \underline{v} \mathrm{~d} \Omega-\int_{\partial_{2} \Omega} \underline{F}_{\mathrm{d}} \cdot \underline{v} \mathrm{~d} S=0, \quad \forall \underline{v} \in \mathcal{U}_{0}, \quad \forall t \in[0, T]\right\}
$$

where we have used the condensed notations $\epsilon(\underline{v})=\frac{1}{2}\left(\nabla \underline{v}+(\nabla \underline{v})^{t}\right)$ and

$$
U_{0}=\left\{\underline{v} \in \mathscr{U}: \underline{v}=0 \text { on } \partial_{1} \Omega\right\}
$$

\subsection{Constitutive relation for plastic and viscoplastic material}

The state of the material is characterized at each point by the total strain $\epsilon$, the (visco)plastic strain $\epsilon^{\mathrm{p}}$ and a set of additional internal variables denoted by $\boldsymbol{\alpha}$. The associated variables are the quantity $\boldsymbol{\beta}$ for $\boldsymbol{\alpha}$ and the stress $\boldsymbol{\sigma}$ for $\boldsymbol{\epsilon}, \boldsymbol{\epsilon}^{\mathrm{p}}$ and $\boldsymbol{\epsilon}^{\mathrm{e}}$. The expression of the dissipation is given by

$$
\boldsymbol{\sigma}: \dot{\boldsymbol{\epsilon}}^{\mathrm{p}}-\boldsymbol{\beta} \circ \dot{\boldsymbol{\alpha}}
$$

The second term specifies the contribution of $(\boldsymbol{\beta}, \dot{\boldsymbol{\alpha}})$ to the dissipation. If $\boldsymbol{\alpha}$ denotes a column of $\mathbb{R}^{n}$, then $\boldsymbol{\beta}$ is also a column of $\mathbb{R}^{n}$ and we have $\boldsymbol{\beta} \circ \dot{\boldsymbol{\alpha}}=\boldsymbol{\beta}^{t} \dot{\boldsymbol{\alpha}}$. More precisely, two spaces $\boldsymbol{e}$ and $\boldsymbol{f}$ are associated by the dissipation duality pairing:

$$
\begin{array}{ll}
{\left[\begin{array}{l}
\dot{\boldsymbol{\epsilon}}^{\mathrm{p}} \\
-\boldsymbol{\alpha}
\end{array}\right],\left[\begin{array}{l}
\boldsymbol{\sigma} \\
\boldsymbol{\beta}
\end{array}\right]} & \rightarrow \boldsymbol{\sigma}: \dot{\boldsymbol{\epsilon}}^{\mathrm{p}}-\boldsymbol{\beta} \circ \dot{\boldsymbol{\alpha}} \\
\boldsymbol{e} \times \boldsymbol{f} & \rightarrow \mathbb{R}
\end{array}
$$

As we work with the small strains assumption, we also have the additivity relation of the elastic strain rate $\dot{\epsilon}^{\mathrm{e}}$ and the (visco)plastic strain rate $\dot{\boldsymbol{\epsilon}}^{\mathrm{p}}\left(\dot{\boldsymbol{\epsilon}}=\dot{\boldsymbol{\epsilon}}^{\mathrm{e}}+\dot{\boldsymbol{\epsilon}}^{\mathrm{p}}\right)$. The material behavior is described in term of state laws, evolution laws and initial conditions.

\subsubsection{The state laws}

According to the first principle of thermodynamics, a scalar function, called the free energy $\psi$, which depends only on the state variables $\epsilon, \epsilon^{\mathrm{p}}$ and $\boldsymbol{\alpha}$ may be introduced. The following classical modeling assumptions are made

- $\psi$ depends only on the elastic strain $\boldsymbol{\epsilon}^{\mathrm{e}}$ and the internal variables $\boldsymbol{\alpha}$

- elasticity is decoupled: $\psi\left(\boldsymbol{\epsilon}^{\mathrm{e}}, \boldsymbol{\alpha}\right)=\psi_{e}\left(\boldsymbol{\epsilon}^{\mathrm{e}}\right)+\psi_{p}(\boldsymbol{\alpha})$

- elasticity is linear: $\psi_{e}\left(\boldsymbol{\epsilon}^{\mathrm{e}}\right)=\frac{1}{2} \boldsymbol{K} \boldsymbol{\epsilon}^{\mathrm{e}}: \boldsymbol{\epsilon}^{\mathrm{e}}$ where $\boldsymbol{K}$ is Hooke's tensor.

The derivation of $\psi$ yields the state equations

$$
\left\{\begin{array}{l}
\boldsymbol{\sigma}=\partial_{\boldsymbol{\epsilon}^{\mathrm{e}}} \psi\left(\boldsymbol{\epsilon}^{\mathrm{e}}, \boldsymbol{\alpha}\right)=K \boldsymbol{\epsilon}^{\mathrm{e}} \\
\boldsymbol{\beta}=\partial_{\boldsymbol{\alpha}} \psi\left(\boldsymbol{\epsilon}^{\mathrm{e}}, \boldsymbol{\alpha}\right)=l(\boldsymbol{\alpha})
\end{array}\right.
$$

where $\boldsymbol{l}(\boldsymbol{\alpha})$ is the derivative of $\psi_{p}$ with respect to $\boldsymbol{\alpha} . \boldsymbol{K}$ is a positive definite operator and $l(\cdot)$ is supposed to be such that $\left(l\left(\boldsymbol{\alpha}_{1}\right)-l\left(\boldsymbol{\alpha}_{2}\right)\right) \circ\left(\boldsymbol{\alpha}_{1}-\boldsymbol{\alpha}_{2}\right) \geqslant 0$ for all $\boldsymbol{\alpha}_{1}, \boldsymbol{\alpha}_{2}$. Then, the free energy $\psi$ is a convex function.

\subsubsection{The evolution laws}

The second principle of thermodynamics, written as $\boldsymbol{\sigma}: \dot{\boldsymbol{\epsilon}}^{\mathrm{p}}-\boldsymbol{\beta} \circ \dot{\boldsymbol{\alpha}} \geqslant 0$, imposes a constraint on the evolution laws relating $\left(\dot{\boldsymbol{\epsilon}}^{\mathrm{p}},-\dot{\boldsymbol{\alpha}}\right)$ to $(\boldsymbol{\sigma}, \boldsymbol{\beta})$. This law can be written

$$
\left[\begin{array}{l}
\dot{\boldsymbol{\epsilon}}^{\mathrm{p}} \\
-\dot{\boldsymbol{\alpha}}
\end{array}\right]=\boldsymbol{B}\left(\left[\begin{array}{l}
\boldsymbol{\sigma} \\
\boldsymbol{\beta}
\end{array}\right]\right)
$$


where $B$ is an operator relevant to the material. It must be positive to satisfy the second principle of thermodynamics. A typical way to define the operator $\boldsymbol{B}$ is to give a scalar function $\varphi^{*}(\boldsymbol{\sigma}, \boldsymbol{\beta})$, generally convex, called the dissipation potential and to write

$$
\left(\begin{array}{l}
\dot{\boldsymbol{\epsilon}}^{\mathrm{p}} \\
-\dot{\boldsymbol{\alpha}}
\end{array}\right) \in\left(\begin{array}{l}
\partial_{\boldsymbol{\sigma}} \varphi^{*}(\boldsymbol{\sigma}, \boldsymbol{\beta}) \\
\partial_{\boldsymbol{\beta}} \varphi^{*}(\boldsymbol{\sigma}, \boldsymbol{\beta})
\end{array}\right)
$$

where $\left(\partial_{\sigma} \varphi^{*}, \partial_{\beta} \varphi^{*}\right)$ denotes the subdifferential of $\varphi^{*}$ at $(\boldsymbol{\sigma}, \boldsymbol{\beta})$. This defines a standard material. When the potential is differentiable, the subdifferential becomes a classical gradient and the belonging an equality. The interest of a standard model lies in the following classical property. The second principle of thermodynamics is fulfilled if the potential satisfies

$$
\varphi^{*} \text { convex, } \quad \varphi^{*}(0,0)=0, \quad \varphi^{*}(\cdot, \cdot) \geqslant 0
$$

Concerning the initial conditions, we assume that the material is virgin at $t=0$ :

$$
\epsilon^{\mathrm{p}}=0, \boldsymbol{\alpha}=0 \text { at } t=0
$$

Finally, note that by eliminating the internal variables in the state laws and evolution laws, one can always, at least formally, associate a stress-strain functional law to an internal variable formulation.

\subsubsection{Example: The Prandtl-Reuss model}

As an example of plastic material behavior described by internal variables, consider the Prandtl-Reuss model. In addition to the plastic strain, the model involves another scalar internal variable $p$ that can be interpreted as the cumulative plastic strain. The free energy is of the form

$$
\psi\left(\epsilon^{\mathrm{e}}, p\right)=\frac{1}{2} K \epsilon^{\mathrm{e}}: \epsilon^{\mathrm{e}}+g(p)
$$

where $g$ is a function characterizing the hardening law. By derivation, we obtain the state laws

$$
\begin{aligned}
& \boldsymbol{\sigma}=\partial \boldsymbol{\epsilon}^{\mathrm{e}} \psi\left(\boldsymbol{\epsilon}^{\mathrm{e}}, p\right)=\boldsymbol{K} \boldsymbol{\epsilon}^{\mathrm{e}} \\
& R=\partial_{p} \psi\left(\boldsymbol{\epsilon}^{\mathrm{e}}, p\right)=g^{\prime}(p)
\end{aligned}
$$

Relation (11) defines the variable $R$, the associate variable to $p$. Classical hardening laws are linear, power and exponential:

$$
R=k_{y} p, \quad R=k_{y} p^{1 / m}, \quad R=R_{M}(1-\exp (-\gamma p))
$$

$k_{y}, m, R_{M}$ and $\gamma$ are strictly positive constant material parameters; then $g$ and $\psi$ are convex functions. Prandtl-Reuss materials are standard with a dissipation potential given by

$$
\varphi^{*}(\boldsymbol{\sigma}, R)=\Psi_{C_{\mathrm{p}}}(\boldsymbol{\sigma}, R)
$$

where we have used the notation $\Psi_{A}(a)$ for the indicator function associated to the convex domain $A$ :

$$
\Psi_{A}(a)=\left\{\begin{array}{lll}
0 & \text { if } & a \in A \\
+\infty & \text { if } & a \notin A
\end{array}\right.
$$

and

$$
C_{\mathrm{p}}=\left\{(\boldsymbol{\sigma}, R) \in f\left\|\boldsymbol{\sigma}^{\mathrm{D}}\right\|-\left(R+R_{0}\right) \leqslant 0, R \geqslant 0\right\}
$$

$\boldsymbol{\sigma}^{\mathrm{D}}$ is the deviatoric part of the stress tensor, $\left\|\boldsymbol{\sigma}^{\mathrm{D}}\right\|=\left(\boldsymbol{\sigma}^{\mathrm{D}}: \boldsymbol{\sigma}^{\mathrm{D}}\right)^{1 / 2}$ and $R_{0}$ is the initial yield stress. The introduction of the condition $R \geqslant 0$ is explained in [33]. A viscoplastic version of the Prandtl-Reuss plastic model is obtained by regularizing the plastic potential. For instance, using a power law type regularization, the potential reads

$$
\varphi^{*}(\boldsymbol{\sigma}, R)=\frac{k_{\mathrm{v}}}{n_{\mathrm{v}}+1}\left\langle\left\|\boldsymbol{\sigma}^{\mathrm{D}}\right\|-\left(R+R_{0}\right)\right\rangle_{+}^{n_{\mathrm{v}}+1}+\Psi_{C_{\mathrm{v}}}(\boldsymbol{\sigma}, R)
$$


where

$$
C_{\mathrm{v}}=\{(\boldsymbol{\sigma}, R) \in f, R \geqslant 0\}
$$

$k_{v}, n_{v}$ are strictly positive constant materials parameters and $\langle a\rangle_{+}$denotes the positive part of $a:\langle a\rangle_{+}=(a+$ $|a|) / 2$.

\subsubsection{Dual potentials}

As indicated earlier, in the standard case, the evolution laws are described in terms of the subdifferential of the dissipation potential, see (6). As a matter of fact, we could also write the evolution in terms of the dual potential $\varphi\left(\dot{\boldsymbol{\epsilon}}^{\mathrm{p}},-\dot{\boldsymbol{\alpha}}\right)$ as

$$
\left(\begin{array}{c}
\boldsymbol{\sigma} \\
\boldsymbol{\beta}
\end{array}\right) \in\left(\begin{array}{c}
\partial_{\dot{\boldsymbol{\epsilon}}^{\mathrm{p}}} \varphi\left(\dot{\boldsymbol{\epsilon}}^{\mathrm{p}},-\dot{\boldsymbol{\alpha}}\right) \\
\partial_{-\dot{\boldsymbol{\alpha}}} \varphi\left(\dot{\boldsymbol{\epsilon}}^{\mathrm{p}},-\dot{\boldsymbol{\alpha}}\right)
\end{array}\right)
$$

The potentials $\varphi^{*}(\boldsymbol{\sigma}, \boldsymbol{\beta})$ and $\varphi\left(\dot{\boldsymbol{\epsilon}}^{\mathrm{p}},-\dot{\boldsymbol{\alpha}}\right)$ are linked through the Legendre-Fenchel transform. See Appendix A for a summary of the properties associated to this transformation. For instance, the dual potential associated to (13) is given by

$$
\varphi\left(\dot{\boldsymbol{\epsilon}}^{\mathrm{p}},-\dot{p}\right)=R_{0}\left\|\dot{\boldsymbol{\epsilon}}^{\mathrm{p}}\right\|+\Psi_{C}\left(\dot{\boldsymbol{\epsilon}}^{\mathrm{p}},-\dot{p}\right)
$$

where

$$
C=\left\{\left(\dot{\boldsymbol{\epsilon}}^{\mathrm{p}},-\dot{p}\right) \in \boldsymbol{e} \mid \operatorname{Tr}\left[\dot{\boldsymbol{\epsilon}}^{\mathrm{p}}\right]=0,\left\|\dot{\boldsymbol{\epsilon}}^{\mathrm{p}}\right\|-\dot{p} \leqslant 0\right\}
$$

and the dual potential to (16) is given by

$$
\varphi\left(\dot{\boldsymbol{\epsilon}}^{\mathrm{p}},-\dot{p}\right)=R_{0}\left\|\dot{\boldsymbol{\epsilon}}^{\mathrm{p}}\right\|+\frac{k_{\mathrm{v}}}{n_{\mathrm{v}}^{\prime}+1}\left(\frac{\left\|\dot{\boldsymbol{\epsilon}}^{\mathrm{p}}\right\|}{k_{\mathrm{v}}}\right)^{n_{\mathrm{v}}{ }^{\prime}+1}+\Psi_{\mathrm{c}}\left(\dot{\boldsymbol{\epsilon}}^{\mathrm{p}},-\dot{p}\right)
$$

where $n_{v}^{\prime}$ is the inverse of $n_{v}$.

Similarly, the state laws (4) may be written in the dual form

$$
\left\{\begin{array}{l}
\epsilon^{\mathfrak{e}}=\partial_{\sigma} \psi^{*}(\boldsymbol{\sigma}, \boldsymbol{\beta}) \\
\boldsymbol{\alpha}=\partial_{\boldsymbol{\beta}} \psi^{*}(\boldsymbol{\sigma}, \boldsymbol{\beta})
\end{array}\right.
$$

For instance, the dual potential to (9) is

$$
\psi^{*}(\boldsymbol{\sigma}, R)=\frac{1}{2} \boldsymbol{K}^{-1} \boldsymbol{\sigma}: \boldsymbol{\sigma}+g^{*}(R)
$$

where $g^{*}(R)$ is the Legendre-Fenchel transform of $g(p)$.

\subsection{Constitutive relation for softening material}

We shall first consider elasticity coupled to isotropic damage. More complex damage models and coupling with (visco)plasticity will be described later.

\subsubsection{Elasticity coupled to isotropic damage}

Let us consider the simple elastic model with softening described below.

$$
\left\{\begin{array}{l}
\boldsymbol{\sigma}=(1-d) \boldsymbol{K}_{0} \epsilon, \quad 0 \leqslant d \leqslant 1 \\
Y=\frac{1}{2} \boldsymbol{K}_{0} \epsilon: \epsilon \\
\dot{d}=h^{\prime}\left(<Y-Y_{0}-\omega(d)>_{+}\right)
\end{array}\right.
$$

where

$$
h^{\prime}(0)=0, \omega(0)=0, \quad h^{\prime}(x) \geqslant 0, \omega^{\prime}(x) \geqslant 0, \quad \forall x \geqslant 0
$$


The scalar $d$ is the damage variable whose value must stay between 0 (non-damaged material) and 1 (completely damaged material). $\boldsymbol{K}_{0}$ is the elastic stiffness of the non-damaged material. It is a positive definite symmetric tensor. The evolution of $d$ is driven by the strain energy $Y$ through the positive material function $h^{\prime}$. The damage may only grow (irreversible phenomena). The material parameter $Y_{0}$ is the threshold under which $d$ may not grow. As the damage proceeds, this threshold rises through the scalar material function $\omega(\cdot)$ which introduces a 'hardening' effect. Characterizing the 'hardening' effect by the additional internal variable $\alpha$ and denoting its associate variable by $\beta$, we may rewrite an equivalent form to the model (24) in which we distinguish the state laws

$$
\left\{\begin{array}{l}
\sigma=(1-d) K_{0} \epsilon, \quad d \leqslant 1 \\
Y=\frac{1}{2} K_{0} \epsilon: \epsilon \\
\beta=\omega(\alpha)
\end{array}\right.
$$

from the evolution laws

$$
\left\{\begin{array}{l}
\dot{d}=h^{\prime}\left(<Y-Y_{0}-\beta>_{+}\right) \\
-\dot{\alpha}=-h^{\prime}\left(<Y-Y_{0}-\beta>_{+}\right)
\end{array}\right.
$$

and the initial conditions:

$$
d=\alpha=0 \quad \text { at } t=0
$$

Choosing $(\epsilon, d, \alpha)$ as independent variables, we were unable to come up with a convex free energy potential $\psi(\epsilon, d, \alpha)$ such that the state laws (26) may be written in the form

$$
\left\{\begin{array}{l}
\boldsymbol{\sigma} \in \partial_{\epsilon} \psi(\epsilon, d, \alpha) \\
Y \in \partial_{d} \psi(\epsilon, d, \alpha) \\
\beta \in \partial_{\alpha} \psi(\epsilon, d, \alpha)
\end{array}\right.
$$

On the other hand, by choosing $(\sigma, d, \alpha)$ as the independent variables, it is possible to prove:

PROPERTY 1. If $\gamma(\cdot)$ is a convex function and $\boldsymbol{K}_{0}$ is a positive definite operator, the free energy given by

$$
(\boldsymbol{\sigma}, d, \alpha) \mapsto \psi(\boldsymbol{\sigma}, d, \alpha)=\frac{1}{2} \frac{\boldsymbol{K}_{0}^{-1} \boldsymbol{\sigma}: \boldsymbol{\sigma}}{(1-d)}+\Psi_{d \leqslant 1}(d)+\gamma(\alpha)
$$

is a convex function.

PROOF. $\psi(\sigma, d, \alpha)$ is of the form $\psi_{\mathrm{d}}(\sigma, d)+\gamma(\alpha) . \gamma(\alpha)$ being convex, we need to prove the convexity of $\psi_{\mathrm{d}}(\boldsymbol{\sigma}, d)$

$$
\psi_{\mathrm{d}}(\boldsymbol{\sigma}, d)=\frac{1}{2} \frac{\boldsymbol{K}_{0}^{-1} \boldsymbol{\sigma}: \boldsymbol{\sigma}}{(1-d)}+\Psi_{d \leq 1}(d)
$$

For $d<1$, The second differential of $\psi_{\mathrm{d}}$ is

$$
\begin{aligned}
\delta^{2} \psi_{\mathrm{d}} & =\frac{\boldsymbol{K}_{0}^{-1} \delta \boldsymbol{\sigma}: \delta \boldsymbol{\sigma}}{(1-d)}+2 \frac{\boldsymbol{K}_{0}^{-1} \boldsymbol{\sigma}: \delta \boldsymbol{\sigma}}{(1-d)^{2}} \delta d+\frac{\boldsymbol{K}_{\bullet}^{-1} \boldsymbol{\sigma}: \boldsymbol{\sigma}}{(1-d)^{3}}(\delta d)^{2} \\
& =\frac{1}{(1-d)^{3}} \boldsymbol{K}_{0}^{-1}(\boldsymbol{\sigma} \delta d+(1-d) \delta \boldsymbol{\sigma}):(\boldsymbol{\sigma} \delta d+(1-d) \delta \boldsymbol{\sigma})
\end{aligned}
$$

which is positive since $\boldsymbol{K}_{0}$ is a positive definite operator.

Defining $\gamma(\alpha)$ as $\gamma(\alpha)=\int_{\bullet}^{\star} \omega\left(\alpha^{\prime}\right) d \alpha^{\prime}$, the state laws (26) are expressed by 


$$
\left\{\begin{array}{l}
\epsilon \in \partial_{\sigma} \psi(\sigma, d, \alpha) \\
Y \in \partial_{d} \psi(\sigma, d, \alpha) \\
\beta \in \partial_{\alpha} \psi(\sigma, d, \alpha)
\end{array}\right.
$$

Note that the choice of $(\boldsymbol{\sigma}, d, \alpha)$ as independent variables is natural to model more complex softening mechanisms $[35,36]$. Concerning the evolution laws, the two pairs of variables $(\dot{d},-\dot{\alpha})$ and $(Y, \beta)$ are associated through the dissipation duality pairing

$$
Y \dot{d}-\beta \dot{\alpha}
$$

Defining the dissipation potential as

$$
\varphi^{*}(Y, \beta)=h\left(<Y-Y_{0}-\beta>_{+}\right)
$$

the evolution laws (27) may be written in the standard form

$$
\left\{\begin{array}{l}
\dot{d}=\partial_{Y} \varphi^{*}(Y, \beta) \\
-\dot{\alpha}=\partial_{\beta} \varphi^{*}(Y, \beta)
\end{array}\right.
$$

EXAMPLE. In the numerical experiments, we shall consider the following expressions for the free energy $\psi(\sigma, d, \alpha)$ and the dissipation potential $\varphi^{*}(Y, \beta)$

$$
\begin{aligned}
& \psi(\boldsymbol{\sigma}, d, \alpha)=\frac{1}{2} \frac{\boldsymbol{K}_{0}^{-1} \boldsymbol{\sigma}: \boldsymbol{\sigma}}{(1-d)}+\Psi_{d \leqslant 1}(d)+Y_{c} \frac{|\alpha|^{3}}{3} \\
& \varphi^{*}(Y, \beta)=\frac{k}{a}<Y-Y_{0}-\beta>_{+}+\frac{k}{a^{2}}\left(\exp \left(-a<Y-Y_{0}-\beta>_{+}\right)-1\right)+\Psi_{\beta \geqslant 0}(\beta)
\end{aligned}
$$

where $Y_{\mathrm{c}}, k, a$ are strictly positive material parameters. The condition $\beta \geqslant 0$ is introduced for the same reason as the introduction of $R \geqslant 0$ in (13), see [33]. The corresponding dual potentials are $\psi^{*}(\epsilon, Y, \beta)$ and $\varphi(\dot{d},-\dot{\alpha})$

$$
\begin{aligned}
& \psi^{*}(\boldsymbol{\epsilon}, Y, \beta)=Y+\Psi_{C_{\mathrm{d}}}(\boldsymbol{\epsilon}, Y)+\frac{2}{3} Y_{\mathrm{c}}\left(\frac{|\beta|}{Y_{\mathrm{c}}}\right)^{3 / 2} \\
& \varphi(\dot{d},-\dot{\alpha})=Y_{0} \dot{d}+\Psi_{C *}(\dot{d},-\dot{\alpha})+\frac{k}{a^{2}} \ln \left(1-\frac{a}{k} \dot{d}\right)+\frac{\dot{d}}{a}\left(1-\ln \left(1-\frac{a}{k} \dot{d}\right)\right)
\end{aligned}
$$

where the convex sets $C_{\mathrm{d}}$ and $C_{\mathrm{d}}^{*}$ are given by

$$
\begin{aligned}
& C_{\mathrm{d}}=\left\{(\epsilon, Y): \frac{1}{2} \boldsymbol{K}_{0} \epsilon: \epsilon-Y \leqslant 0\right\} \\
& C_{\mathrm{d}}^{*}=\{(\dot{d},-\dot{\alpha}): 0 \leqslant \dot{d} \leqslant k / a, \dot{d}-\dot{\alpha} \leqslant 0\}
\end{aligned}
$$

\subsubsection{More complex damage model}

In the case of anisotropic damage, the damage need to be expressed in a more complex manner than with a scalar $d$, for instance by a vector $\underline{d}$. The free energy in terms of the variables $(\sigma, \underline{d}, \alpha)$ takes now the general form

$$
\psi(\boldsymbol{\sigma}, \underline{d}, \alpha)=\frac{1}{2}\left(K(\underline{d})^{-1}\right) \boldsymbol{\sigma}: \boldsymbol{\sigma}+\Psi_{b}(\underline{d})+\gamma(\alpha)
$$

where $\Psi_{D}(\underline{d})$ is a generalization of $\Psi_{d \leqslant 1}(d)$. D is the convex set of admissible damage vector $\underline{d}$ defined by

$$
D=\{d: K(\underline{d}) \text { is a positive operator }\}
$$

Concerning the convexity of the free energy function (37), we have the following property.

PROPERTY 2. If the relation $\underline{d} \mapsto \boldsymbol{K}(\underline{d})$ is linear and such that $\boldsymbol{K}(\underline{0})=\boldsymbol{K}_{\mathbf{0}}$ is a positive definite operator and, if $\gamma(\cdot)$ is a convex function, then, the free energy given by 


$$
(\boldsymbol{\sigma}, \underline{d}, \alpha) \mapsto \psi(\boldsymbol{\sigma}, \underline{d}, \alpha)=\frac{1}{2}\left(\boldsymbol{K}(\underline{d})^{-1}\right) \boldsymbol{\sigma}: \boldsymbol{\sigma}+\Psi_{\mathbf{p}}(\underline{d})+\gamma(\alpha)
$$

is a convex function.

PROOF. The second differential of $\frac{1}{2}\left(K(\underline{d})^{-1}\right) \boldsymbol{\sigma}: \boldsymbol{\sigma}$ is

$$
\begin{array}{r}
\delta^{2}\left(\frac{1}{2}\left(\boldsymbol{K}(\underline{d})^{-1} \boldsymbol{\sigma}: \boldsymbol{\sigma}\right)=\boldsymbol{K}^{-1} \delta \boldsymbol{\sigma}: \delta \boldsymbol{\sigma}+\boldsymbol{K}^{-1}\left(\delta \boldsymbol{K} \boldsymbol{K}^{-1} \boldsymbol{\sigma}\right):\left(\delta \boldsymbol{K} \boldsymbol{K}^{-1} \boldsymbol{\sigma}\right)-2 \boldsymbol{K}^{-1}\left(\delta \boldsymbol{K} \boldsymbol{K}^{-1} \boldsymbol{\sigma}\right): \delta \boldsymbol{\sigma}-\right. \\
\frac{1}{2} \delta^{2} \boldsymbol{K}\left(\boldsymbol{K}^{-1} \boldsymbol{\sigma}\right):\left(\boldsymbol{K}^{-1} \boldsymbol{\sigma}\right)=\boldsymbol{K}^{-1}\left(\delta \boldsymbol{\sigma}-\delta \boldsymbol{K} \boldsymbol{K}^{-1} \boldsymbol{\sigma}\right):\left(\delta \boldsymbol{\sigma}-\delta \boldsymbol{K} \boldsymbol{K}^{-1} \boldsymbol{\sigma}\right)-\frac{1}{2} \delta^{2} \boldsymbol{K}\left(\boldsymbol{K}^{-1} \boldsymbol{\sigma}\right):\left(\boldsymbol{K}^{-1} \boldsymbol{\sigma}\right)
\end{array}
$$

Since $K^{-1}$ is positive for $\underline{d} \in D$, the convexity of $\psi$ is insured if

$$
-\frac{1}{2} \delta^{2} K\left(K^{-1} \boldsymbol{\sigma}\right):\left(K^{-1} \boldsymbol{\sigma}\right) \geqslant 0
$$

which is true if $\underline{d} \mapsto K(\underline{d})$ is linear since $\delta^{2} K=0$.

\subsubsection{Viscoplasticity coupled to damage}

Concerning the evolution laws, a natural way to couple (visco)plasticity and damage is to introduce the effective quantities $\tilde{\boldsymbol{\sigma}}, \tilde{\boldsymbol{\epsilon}}^{\mathrm{p}}$ (see $\left.[35,36]\right)$

$$
\tilde{\boldsymbol{\sigma}}=\frac{\boldsymbol{\sigma}}{(1-d)}, \quad \tilde{\boldsymbol{\epsilon}}^{\mathrm{p}}=\dot{\boldsymbol{\epsilon}}^{\mathrm{p}}(1-d)
$$

and to write the viscoplastic part of the model in terms of these new quantities instead of the classical ones $\boldsymbol{\sigma}$, $\epsilon^{\mathrm{p}}, \dot{\epsilon}^{\mathrm{p}}$.For instance, for the Prandtl-Reuss model described in Section 2.2 coupled to the isotropic, the two set of variables involved in the duality pairing are $\left(\tilde{\boldsymbol{\epsilon}}^{\mathrm{p}},-\tilde{\dot{p}}, \dot{d},-\dot{\alpha}\right)$ and $(\tilde{\boldsymbol{\sigma}}, \tilde{R}, Y, \beta)$. The dissipation reads

$$
\tilde{\boldsymbol{\sigma}}: \tilde{\boldsymbol{\epsilon}}^{p}-\tilde{R} \tilde{p}+Y \dot{d}-\beta \dot{\alpha}
$$

and the potential of dissipation is

$$
\varphi^{*}(\tilde{\boldsymbol{\sigma}}, \tilde{R}, Y, \beta)=\varphi_{1}^{*}(\tilde{\boldsymbol{\sigma}}, \tilde{R})+\varphi_{2}^{*}(Y, \beta)
$$

where $\varphi_{1}^{*}(\tilde{\boldsymbol{\sigma}}, \tilde{R})$ is given by $(13)$ in plasticity and (16) in viscoplasticity, $(\boldsymbol{\sigma}, R)$ being replaced by $(\tilde{\boldsymbol{\sigma}}, \tilde{R})$, and $\varphi_{2}^{*}(Y, \beta)$ is given by $(34)$.

Concerning the state laws, the following internal variables are involved: $\boldsymbol{\sigma}, d, \alpha$ and $\tilde{p}$. The free energy reads

$$
\psi(\boldsymbol{\sigma}, d, \alpha, \tilde{p})=\frac{1}{2} \frac{\boldsymbol{K}_{0}^{-1} \boldsymbol{\sigma}: \boldsymbol{\sigma}}{(1-d)}+\Psi_{d \leqslant 1}(d)+\gamma(\alpha)+g(\tilde{p})
$$

and the associated variable to $\tilde{p}$ is $\tilde{R}$.

\section{A new constitutive error relation family}

The notion of error in the constitutive law has been introduced in [37]. The general concept is to split the equations of the problem into two groups with different mechanical contents. The first group involves 'safe' equations like the kinematic and static constraints whereas the second group involves less reliable equations: constitutive relation related equations. A solution satisfying the first group is called admissible. An admissible solution is the exact solution to the problem if and only if it satisfies the equations of the second group. Thus the quality of the admissible solution may be evaluated through the residuals associated to the equations of the second group. Since the second group is related to the constitutive relation, the terminology of error in the constitutive law was naturally introduced. 
The splitting of the equations into the two groups is not always obvious. For instance, when the material behavior is described using internal variables, the state laws may be seen as 'internal' equilibrium relations and thus included in the first group, or, seen as constitutive law like the evolution laws and thus included in the second group. The first choice was introduced in $[31,32]$ and investigated in $[30,33,34]$. This choice leads to the terminology of dissipation error since the error is measured through the residuals of the sole evolution laws, the state laws being a priori satisfied.

Here we wish to investigate the second choice, i.e. consider the state laws as part of the second group. The error now involves the residuals associated to both the state and evolution laws. We shall see that this second choice allows to fit elasticity, plasticity, viscoplasticity with or without softening in the same framework. The dissipation error is recovered as a particular case by enforcing strongly the state laws.

\subsection{Admissible solution}

The model problem for a structure exhibiting (visco)plastic behavior with or without softening involve the following equations (Section 2):

- the kinematic and static constraints,

$$
\underline{u} \in \mathcal{U}_{a d}^{[0, T]}, \quad \epsilon \in \mathscr{E}_{a d}^{[0, T]}, \quad \boldsymbol{\sigma} \in \mathscr{S}_{a d}^{[0, T]}
$$

- the state laws;

- the evolution laws;

- the additivity of the elastic and (visco)plastic strain rates: $\dot{\boldsymbol{\epsilon}}=\dot{\boldsymbol{\epsilon}}^{\mathrm{e}}+\dot{\boldsymbol{\epsilon}}^{\mathrm{p}}$

- the initial conditions on the internal variables.

We split these equations into two groups. The first group, which defines the admissibility of a solution, gathers the kinematic and static constraints, the additivity relation and the initial conditions. The second group on which the quality of the admissible solution will be evaluated involves the remaining equations, i.e. the state laws and evolution laws. It is quite natural to put the relation $\dot{\boldsymbol{\epsilon}}=\dot{\boldsymbol{\epsilon}}^{\mathrm{e}}+\dot{\boldsymbol{\epsilon}}^{\mathrm{p}}$ in the first group since this relation is independent of the state and evolution laws and may be seen as an 'internal' kinematic constraint.

\subsection{Definition of the error}

The error measures the non-satisfaction of the state laws and evolution laws by the admissible solution. Since these laws are expressed in terms of a potential: free energy for the state laws and dissipation potential for the evolution laws, we may use the property of the Legendre-Fenchel, equations (A.5)-(A.6), to compute the residuals on these laws. For instance, in the case of the Prandtl-Reuss (visco)plastic model coupled to isotropic damage, the residual on the state laws is based on the quantity

$$
\psi^{*}\left(\epsilon^{\mathrm{e}}, Y, \beta, \tilde{R}\right)+\psi(\boldsymbol{\sigma}, d, \alpha, \tilde{p})-\boldsymbol{\sigma}: \epsilon^{\mathrm{e}}-Y d-\beta \alpha-\tilde{R} \tilde{p}
$$

that we shall denote by

$$
\eta_{\psi}\left(\epsilon^{\mathrm{e}}, Y, \beta, \tilde{R} ; \boldsymbol{\sigma}, d, \alpha, \tilde{p}\right)
$$

$\psi^{*}$ and $\psi$ are dual potentials and their expressions depend on the specific law chosen, see Section 2 . Concerning the evolution laws, the residual is based on the quantity

$$
\varphi^{*}(\tilde{\boldsymbol{\sigma}}, \tilde{R}, Y, \beta)+\varphi\left(\tilde{\boldsymbol{\epsilon}}^{\mathrm{p}},-\overline{\dot{p}}, \dot{d}, \dot{\alpha}\right)-\tilde{\boldsymbol{\sigma}} \tilde{\tilde{\boldsymbol{\epsilon}}}^{\mathrm{p}}+\tilde{R} \dot{p}-Y \dot{d}+\beta \dot{\alpha}
$$

or shortly

$$
\eta_{\varphi}\left(\tilde{\boldsymbol{\sigma}}, \tilde{R}, Y, \beta ; \tilde{\boldsymbol{\epsilon}}^{\mathrm{p}},-\tilde{\dot{p}}, \dot{d},-\dot{\alpha}\right)
$$

The definition of $\tilde{\boldsymbol{\sigma}}$ and $\tilde{\boldsymbol{\epsilon}}^{\mathrm{p}}$ are given by (41).

For the admissible solution to be the exact solution, the following residual 


$$
e_{x, t}^{2}=\left.\eta_{\psi}\left(\boldsymbol{\epsilon}^{\mathrm{e}}, Y, \beta, \tilde{R} ; \boldsymbol{\sigma}, d, \alpha, \tilde{p}\right)\right|_{t}+\int_{0}^{t} \eta_{\varphi}\left(\tilde{\boldsymbol{\sigma}}, \tilde{R}, Y, \beta ; \tilde{\boldsymbol{\epsilon}}^{\mathrm{p}},-\tilde{p}, \dot{d},-\dot{\alpha}\right) \mathrm{d} t
$$

must be zero for all $x \in \Omega$ and for all $t \in[0, T]$. To define a global error in time and space, we globalize the residual (50). First, we integrate over the space, see (51); then, we define the contribution to the error over $[0, t]$, see (52); and, finally, we define the absolute error $e$ by taking the supremum over the time, see (53).

$$
\begin{aligned}
& e_{t}^{2}=\int_{\Omega} e_{x, t}^{2} \mathrm{~d} \Omega \\
& e_{[0, t]}^{2}=\sup _{\tau \in[0, t]} e_{\tau}^{2} \\
& e^{2}=\sup _{t \in[0, T]} e_{[0, t]}^{2}
\end{aligned}
$$

The relative error, $\epsilon$, is defined by

$$
\epsilon^{2}=\frac{e^{2}}{\sup _{i \in[0, T]} d_{t}^{2}}
$$

where

$$
d_{t}^{2}=\int_{\Omega} \psi^{*}\left(\boldsymbol{\epsilon}^{\mathrm{c}}, Y, \beta, \tilde{R}\right)+\left.\psi(\boldsymbol{\sigma}, d, \alpha, \tilde{p}) \mathrm{d} \Omega\right|_{t}+\int_{0}^{t} \int_{\Omega} \psi^{*}(\tilde{\boldsymbol{\sigma}}, \tilde{R}, Y, \beta)+\psi\left(\tilde{\boldsymbol{\epsilon}}^{\mathrm{p}},-\tilde{p}, \dot{d},-\dot{\alpha}\right) \mathrm{d} \Omega \mathrm{d} t
$$

REMARKS. The expressions (50) and (55), involve both state laws and evolution laws related quantities. We have implicitly chosen to give an equal weight to these two influences. Of course, one can introduce a different weighting. When some zones in the domain $\Omega$ are fully damaged ( $d=1$ or $\underline{d} \notin D$ ), they are removed from the space integration appearing in (51).

\subsection{Particular cases}

In the case of pure elasticity reference, the error defined above coincide with the classical error in the constitutive law for elasticity $[2,38]$. Indeed, for pure elasticity, the strain coincide with the elastic strain and the (visco)plastic strain is zero. The evolution laws do not appear in the model and the free energy involves only the elastic energy. Thus, the relative error $\epsilon$ defined above reduces to

$$
\epsilon^{2}=\frac{\sup _{t \in[0, T]} \int_{\Omega} \frac{1}{2} K \epsilon: \epsilon+\frac{1}{2} K^{-1} \sigma: \sigma-\sigma:\left.\epsilon \mathrm{d} \Omega\right|_{t}}{\sup _{t \in[0 . T]} \int_{\Omega} \frac{1}{2} K \epsilon: \epsilon+\frac{1}{2} K^{-1} \sigma:\left.\sigma \mathrm{d} \Omega\right|_{t}}
$$

Assuming a radial loading, we obtain

$$
\epsilon^{2}=\frac{\int_{\Omega} \frac{1}{2} K \epsilon: \epsilon+\frac{1}{2} K^{-1} \sigma: \sigma-\sigma:\left.\epsilon \mathrm{d} \Omega\right|_{T}}{\int_{\Omega} \frac{1}{2} K \epsilon: \epsilon+\frac{1}{2} K^{-1} \sigma:\left.\sigma \mathrm{d} \Omega\right|_{T}}=\frac{\int_{\Omega} \frac{1}{2} K^{-1}(\sigma-K \epsilon):\left.(\sigma-K \epsilon) \mathrm{d} \Omega\right|_{T}}{\int_{\Omega} \frac{1}{2} K \epsilon: \epsilon+\frac{1}{2} K^{-1} \sigma:\left.\sigma \mathrm{d} \Omega\right|_{T}}
$$

Another particular case is the one in which the admissible solution satisfies a priori the state laws. The error reduces to

$$
\epsilon^{2}=\frac{\int_{0}^{T} \int_{\Omega} \eta_{\varphi}\left(\tilde{\boldsymbol{\sigma}}, \tilde{R}, Y, \beta ; \tilde{\boldsymbol{\epsilon}}^{\mathrm{p}},-\tilde{p}, \dot{d},-\dot{\alpha}\right) \mathrm{d} \Omega \mathrm{d} t}{\sup _{t \in[0, T]} d_{t}^{2}}
$$

which is similar to the dissipation error [33]. 


\section{Implementation of the errors for the FEM}

We consider the classical incremental finite element method. Let us give the main features of this method. The problem to be solved on $[0, T]$ is divided into a succession of resolutions over $\left[t_{n}, t_{n+1}\right](n=0, \ldots, N$; $t_{0}=0 ; t_{N+1}=T$ ). Assuming the solution is known up until the time instant $t_{n}$, one must then build the solution over $\left[t_{n}, t_{n+1}\right]$. Both time and space discretizations need to be performed. Then, the resulting non-linear algebraic system is solved using an iterative technique.

\subsection{Time discretization}

A common choice is to assume a linear evolution of the solution over $\left[t_{n}, t_{n+1}\right]$ so that the problem reduces to the search of the solution at $t_{n+1}$. The equilibrium equations and kinematic constraints are written at $t_{n+1}$ as well as the decomposition into elastic and (visco)plastic strains:

$$
\epsilon_{n+1}=\epsilon_{n+1}^{\mathrm{e}}+\epsilon_{n+1}^{\mathrm{p}}
$$

The state and evolution laws are discretized in order to link algebraically the value of the field at $t_{n+1}$ and $t_{n}$. For instance, we may impose the state laws to be satisfied at $t_{n+1}$ :

$$
\eta_{\psi}\left(\epsilon_{n+1}^{\mathrm{e}}, Y_{n+1}, \beta_{n+1}, \tilde{R}_{n+1} ; \boldsymbol{\sigma}_{n+1}, d_{n+1}, \alpha_{n+1}, \tilde{p}_{n+1}\right)=0
$$

and we may use a generalized mid-point rule to discretize the evolution laws:

$$
\eta_{\varphi}\left(\tilde{\boldsymbol{\sigma}}_{\theta}, \tilde{R}_{\theta}, Y_{\theta}, \beta_{\theta} ; \tilde{\boldsymbol{\epsilon}}_{n+1}^{\mathrm{p}},-\tilde{\dot{p}}_{n+1}, \dot{d}_{n+1},-\dot{\alpha}_{n+1}\right)=0
$$

where we have used the notations

$$
\dot{x}_{n+1}=\frac{x_{n+1}-x_{n}}{t_{n+1}-t_{n}}, \quad x_{\theta}=(1-\theta) x_{n}+\theta x_{n+1} 0 \leqslant \theta \leqslant 1
$$

\subsection{Space discretization}

Concerning the space discretization, the displacement space, $\mathscr{U}$, is replaced by a finite element subset $\mathcal{U}_{h} \subset \mathcal{U}$. The equilibrium condition appearing in (3) is imposed in the weak sense:

$$
\int_{\Omega_{h}} \tau: \epsilon(\underline{v}) \mathrm{d} \Omega_{h}-\int_{\Omega} f_{\mathrm{d}} \cdot \underline{v} \mathrm{~d} \Omega-\int_{\partial_{2} \Omega} \underline{F_{\mathrm{d}}} \underline{v} \mathrm{~d} S=0 \quad \forall \underline{v} \in \mathscr{U}_{h, 0}
$$

where

$$
\mathscr{U}_{h, 0}=\left\{\underline{v} \in \mathscr{U}_{h} \text { such that } \underline{v}=0 \text { on } \partial_{1} \Omega\right\}
$$

The notation $\Omega_{h}$, instead of $\Omega$, in the first term of (62) expresses the fact that due to the nonlinear constitutive relation, this term cannot be in general integrated exactly and therefore is expressed in term of a numerical integration.

\subsection{Iterative technique}

The system to be solved at each time step is algebraic and nonlinear. This system is solved using an iterative technique, typically Newton's method. As the iterations proceed, two types of solutions must be distinguished: a solution satisfying the discrete kinematic and static equations, and a solution satisfying the discrete kinematic and constitutive relation. When the difference between the two types of solutions is under a given tolerance $\delta_{\text {tol }}$, for instance in the $L^{2}$ norm of the difference in stresses (choice for the numerical experiments to be shown later), the iterative procedure stops and the finite element code generally gives the solution satisfying the discrete constitutive relation. 


\subsection{Construction of an admissible solution}

The error cannot be directly measured on the finite element solution because it is not generally admissible. For instance, the finite element stresses are only in equilibrium in a weak sense. Thus, we need to build an admissible solution from the finite element one. The construction is similar to the one introduced for the dissipation error in [33]. We summarize the procedure below.

- The finite element displacement and strain solutions are kept at each time-step since they are kinematically admissible.

- The finite element stress solution is in equilibrium in a weak sense at each time step. From these stresses, one may build stresses rigourously in equilibrium using equilibration techniques. These techniques were first introduced for elasticity problems [2,3] but may be extended with minor changes to other type of behavior since they are intresically material independent. They were for instance used for plasticity problem in [28].

- The admissible displacement, strain and stress fields built at each time-step are interpolated linearly in between the time-steps. (The loading is assumed piece-wise linear.)

- Concerning the internal and associate variables, a general strategy is to build them such that they minimize the error. In this minimization, the stress and strain field history is known and the total strain must be the sum of the elastic and (visco)plastic strain. In other words for given stress and strain history, we chose the admissible solution minimizing the error. Since the internal variables are not involved in spatial partial differential equation, the minimization may be carried out independently from point to point. For instance, in the case of the Prandtl-Reuss model coupled to isotropic damage, the quantity to minimize is

$$
\sup _{t \in[0, T]}\left(\left.\eta_{\psi}\left(\epsilon^{\mathrm{e}}, Y, \beta, \tilde{R} ; \boldsymbol{\sigma}, d, \alpha, \tilde{p}\right)\right|_{t}+\int_{0}^{t} \eta_{\varphi}\left(\tilde{\boldsymbol{\sigma}}, \tilde{R}, Y, \beta ; \tilde{\boldsymbol{\epsilon}}^{\mathrm{p}},-\tilde{\tilde{p}}, \dot{d},-\dot{\alpha}\right) \mathrm{d} t\right)
$$

The unknowns are the history of $\left(\epsilon^{\mathrm{e}}, Y, \beta, \tilde{R} ; \epsilon^{\mathrm{p}}, d, \alpha, \tilde{p}\right)$ over $[0, T]$ and the constraints are

$\boldsymbol{\sigma}, \boldsymbol{\epsilon}$ given over $[0, T], \epsilon=\epsilon^{\mathrm{e}}+\epsilon^{\mathrm{p}}$ on $[0, T]$, initial conditions satisfied

Practically, we simplify this problem by assuming a piece-wise linear evolution of the unknowns and by minimizing through a step by step strategy: knowing the the admissible solution at $t_{n}$, the problem is to find the admissible solution at $t_{n+1}$ minimizing the function

$$
\sup _{t \in\left[t_{n}, t_{n+1}\right]}\left(\left.\eta_{\psi}\left(\epsilon^{\mathrm{e}}, Y, \beta, \tilde{R} ; \boldsymbol{\sigma}, d, \alpha, \tilde{p}\right)\right|_{\ell}+\int_{t_{n}}^{t} \eta_{\varphi}\left(\tilde{\boldsymbol{\sigma}}, \tilde{R}, Y, \beta ; \tilde{\epsilon}^{\mathrm{p}},-\tilde{p}, \dot{d}, \dot{\alpha}\right) \mathrm{d} t\right)
$$

The unknowns are the values of $\left(\epsilon^{\mathrm{e}}, Y, \beta, \tilde{R} ; \epsilon^{\mathrm{p}}, d, \alpha, \tilde{p}\right)$ at $t_{n+1}$ (linear evolution over $\left.\left[t_{n}, t_{n+1}\right]\right)$ under the constraints: $\sigma_{n+1}, \epsilon_{n+1}$ given and $\epsilon_{n+1}=\epsilon_{n+1}^{\mathrm{e}}+\epsilon_{n+1}^{\mathrm{p}}$.

\section{Numerical experiments}

\subsection{Numerical experiments for (visco)plastic structures without softening}

We shall consider two plane stress problems: a frame under non-monotonic loading and a square plate under volumic loading. The first problem is treated in plasticity: Prandtl-Reuss model with linear hardening whereas the second problem is treated in viscoplasticity (also with a linear hardening). We consider the particular case of the error in which the state laws are enforced a priori, see (57). The precise expression of the error for this case may be found in [33].

The frame in plane stress state, Fig. 1, is submitted on its right side to a growing loading followed by a decreasing loading (thin line, Fig. 2). Then, a growing pressure is applied to its upper part (thick line, Fig. 2). The dimensionless material parameters are

$$
R_{0}=1, \quad k_{y}=8.16, \quad E=244.95, \quad \nu=0.3
$$

$E$ and $\nu$ denote Young's modulus and Poisson's ratio, respectively. The mesh has been optimized in elasticity 


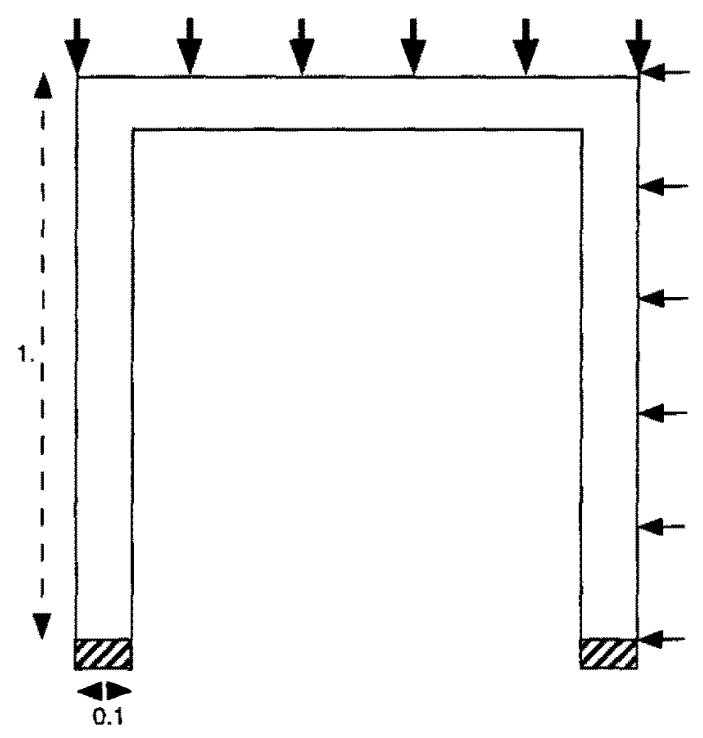

Fig. 1. The geometry and applied loading on the frame.
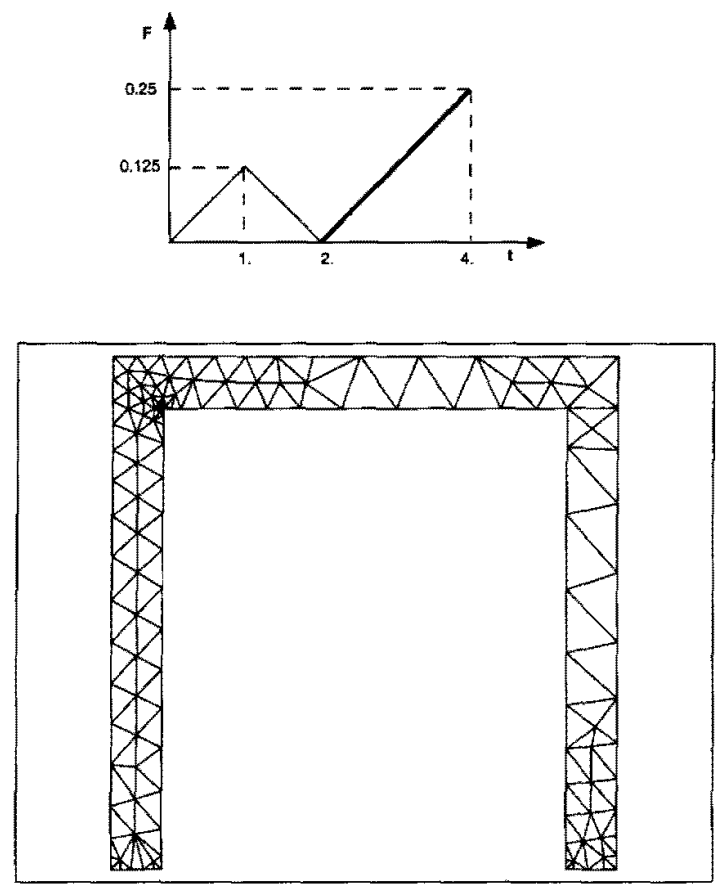

Fig. 2. Evolution of the loading and the mesh: 194 six-node triangles.

for an error of $10 \%$, Fig. 2. It is composed of 194 six-node triangular elements. Three time steps are used and the tolerance for the iterative technique is set to $\delta_{\text {tol }}=10^{-2}$. The dissipation error obtained is $28.15 \%$.

The square plate, Fig. 3, is loaded on its right side under uniform tension. A uniform body force places this tension in equilibrium. Owing to the symmetry, only the upper-half part of the plate is studied (Fig. 3). The loading evolves monotonically (Fig. 4). The dimensionless materials parameters are:

$$
R_{0}=0.7, \quad k_{y}=8.16, \quad E=244.95, \quad \nu=0.3, \quad n_{\mathrm{v}}=2, \quad k_{\mathrm{v}}=2.25
$$

Three time steps are used and the tolerance for the iterative technique is set to $\delta_{\text {tol }}=10^{-6}$. The mesh, Fig. 5 ,

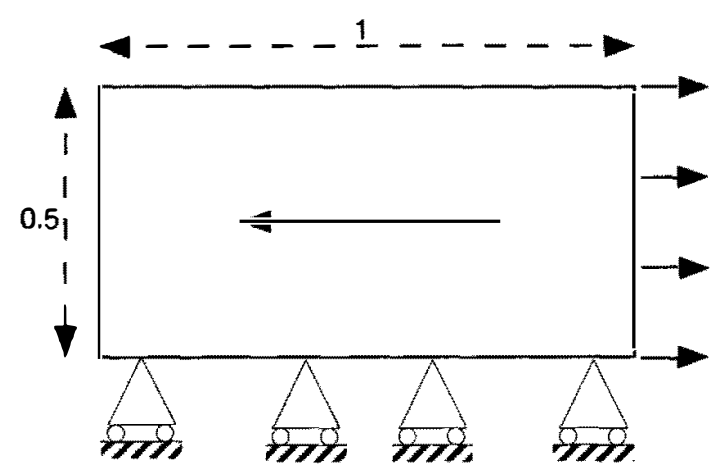

Fig. 3. The square plate problem.

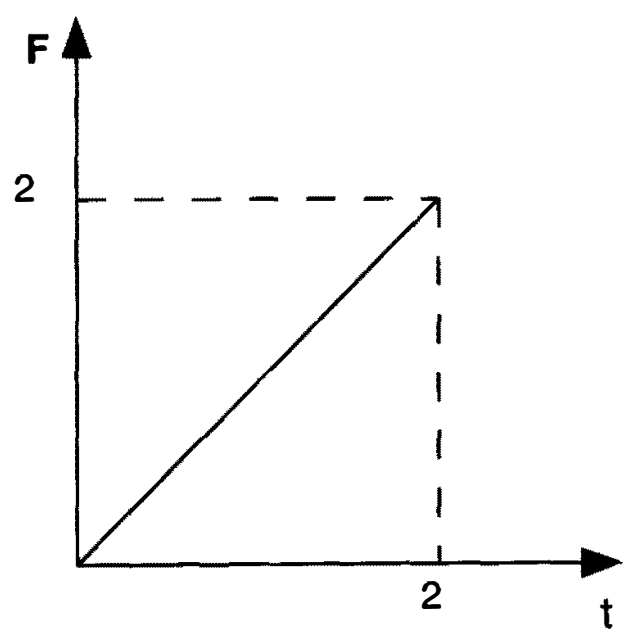

Fig. 4. The loading. 


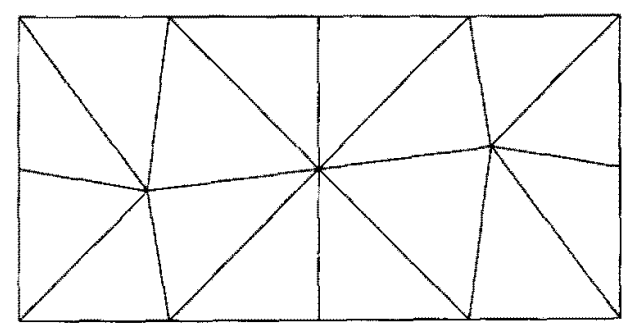

Fig. 5. The mesh: 16 three-node triangles.

contains 16 three-node triangular elements. The plastic computation is carried out with four identical time increments. The error dissipation is $21.30 \%$ for this computation.

Table 1 shows the influence of the numerical parameters on the value of the error. First, for a given time discretization and a given tolerance $\left(\delta_{\text {tol }}=10^{-3}\right)$, we consider a sequence of six meshes with an increasing number of degrees of freedom as the mesh number increases. Second, for a given mesh and tolerance $\left(\delta_{\text {tol }}=10^{-3}\right)$, the number of time steps is increased. Finally, for a given time-space discretization (fine or coarse), the tolerance is reduced. We notice that in each of the three cases, the error drops but finally tends to stabilize. The drop phase in the error evolution suggests that the error depends on the three error sources (mesh, time discretization and iterative technique) through their respective parameter: mesh size, time step size and tolerance. The stabilization phase suggests that the influence of a parameter on the error becomes negligible as it's corresponding error source become negligible in front of the two other error sources. Table 2 summarizes the numerical parameter influences for the square plate problem. The results follow the same trend as in Table 1.

\subsection{First numerical experiments for (visco)plastic structures with softening}

We consider a beam with a flaw, Fig. 6, fixed on its left end and submitted to a growing displacement on its right end, Fig. 7. The length of the beam is 1 (dimensionless treatment of the problem). The behavior of the

Table 1

Influence of the numerical parameters on the error for the frame problem

\begin{tabular}{|c|c|c|c|c|c|c|}
\hline $\begin{array}{l}\text { mesh } \\
\epsilon\end{array}$ & $\begin{array}{r}\text { mesh } 1 \\
40.94\end{array}$ & $\begin{array}{l}\text { mesh2 } \\
28.99\end{array}$ & $\begin{array}{l}\text { mesh3 } \\
25.74\end{array}$ & $\begin{array}{c}\text { mesh4 } \\
23.96\end{array}$ & $\begin{array}{l}\text { mesh5 } \\
20.80\end{array}$ & $\begin{array}{r}\text { mesh6 } \\
18.92\end{array}$ \\
\hline nb steps & 3 & 6 & 12 & 24 & 48 & \\
\hline$\epsilon$ & 25.74 & 16.36 & 13.85 & 13.76 & 14.00 & \\
\hline$\delta_{t 01}$ & $10^{\circ}$ & $10^{-1}$ & $10^{-2}$ & $10^{-3}$ & $10^{-4}$ & $10^{-5}$ \\
\hline$\epsilon$ (coarse) & 26.60 & 16.42 & 16.36 & 16.37 & 16.37 & 16.37 \\
\hline$\epsilon$ (fine) & 12.40 & 6.05 & 5.37 & 5.36 & 5.36 & 5.36 \\
\hline
\end{tabular}

Table 2

Influence of the numerical parameters on the error for the square plate problem

\begin{tabular}{lccccc}
\hline mesh & mesh1 & mesh2 & mesh3 & mesh4 & mesh5 \\
$\epsilon$ & 24.90 & 17.62 & 12.54 & 9.88 & 8.81 \\
\hline nb steps & 1 & & & & 8 \\
$\epsilon$ & 42.25 & 2 & 4 & 13.76 & 16 \\
\hline
\end{tabular}




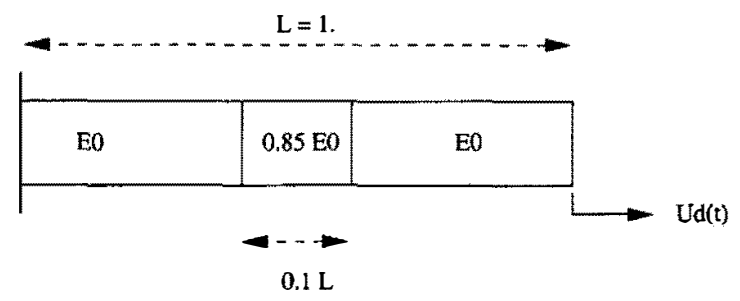

Fig. 6. Geometry and loading for the beam problem.

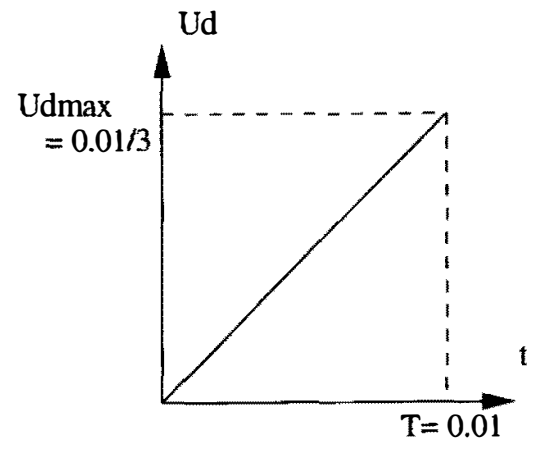

Fig. 7. Evolution of the loading on the beam as a function of time.

beam material is elastoplastic coupled to isotropic damage and follows the equations (24). In fact, the initial yield stress $R_{0}$ is chosen high enough to avoid plasticity. The state laws and the evolution laws derives from the potentials given in (34)-(36). The material parameters are

$$
E_{0}=6350, \quad Y_{0}=0, \quad Y_{\mathrm{c}}=0.13, \quad k=7000, \quad a=1, \quad R_{0}=1000, \quad k_{y}=22582, \quad m=1
$$

where $E_{0}$ is the young modulus of the non damaged material.

Fig. 8 gives the evolution of the damage for a computation carried out with 100 time steps and a uniform mesh of 50 elements. A strong localization of the damage in the flaw region occurs as soon as $d$ reaches 0.5

Fig. 9 shows the space-time map of the local contributions to the error defined in (50). We note than the error is localized in the vicinity of the rupture. The evolution of the contribution to the relative erorr through time is given Fig. 10. This quantity is defined by (52) and normed by the denominator appearing in (54).

Table 3 shows the influence of the numerical parameters on the error. First, for a given space discretization (10 elements) and a given tolerance for the iterative technique $\left(\delta_{\text {tol }}=10^{-10}\right)$, the number of time steps is increased. Second, for a given time-space discretization (10 elements and 100 time-steps), the tolerance is reduced. We observe that in the second case, the error decreases and then stabilizes because the time discretization is becoming the predominant source of error. Provided that the mesh takes into account the boundary of the flaw region, the number of elements has little influence on the error in this problem.

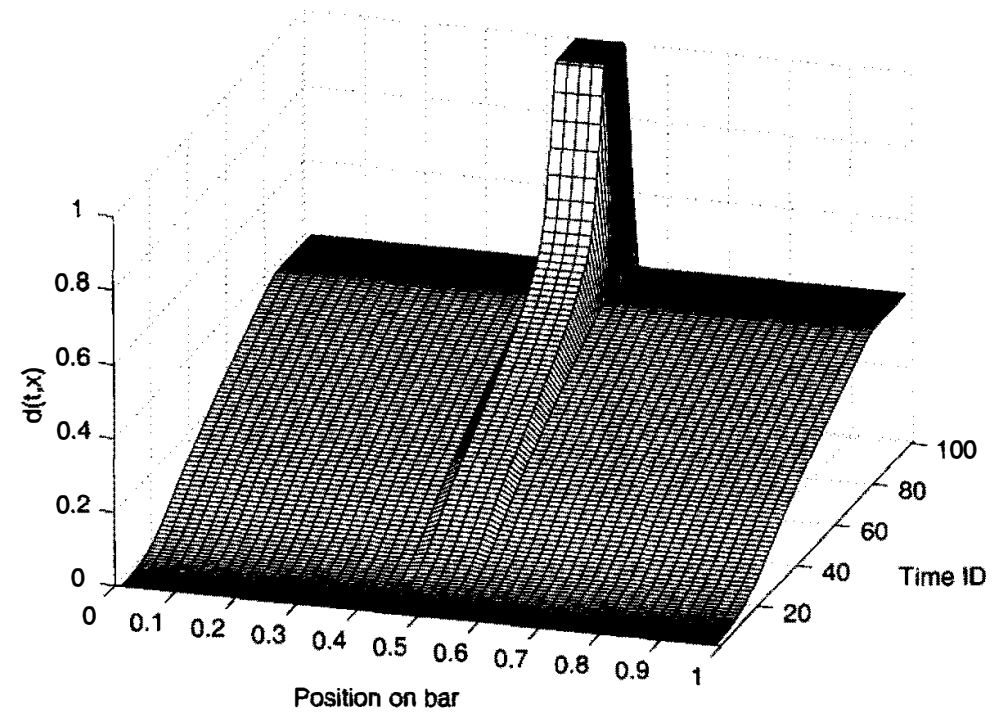

Fig. 8. Damage scalar variable $d$ as a function of the time and space location on the bar. 


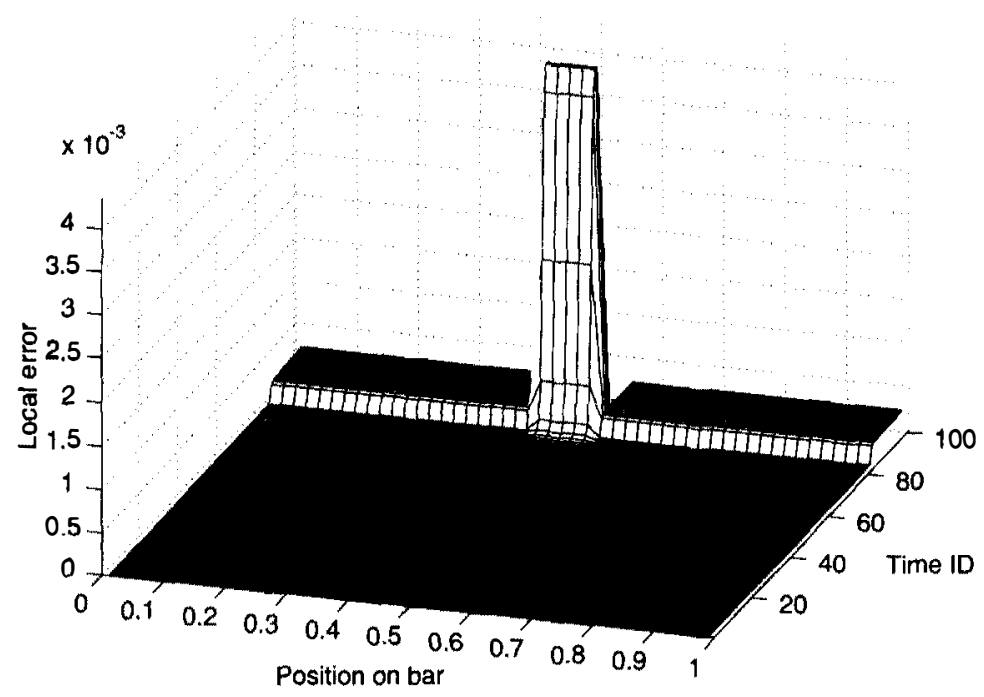

Fig. 9. Local contributions to the error.

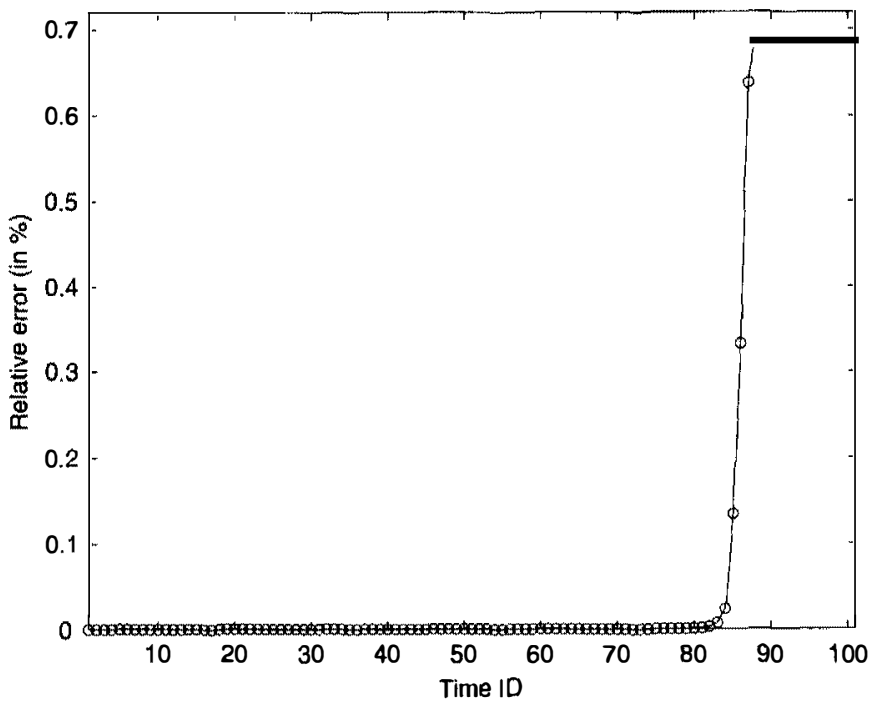

Fig. 10. Contribution to the relative error through time.

Table 3

Influence of the numerical parameters on the error

\begin{tabular}{lllllllll}
\hline nb steps & 2 & 3 & 5 & 7 & 10 & 50 & 100 & 150 \\
$\epsilon(\%)$ & 50.96 & 15.02 & 10.13 & 8.62 & 5.40 & 2.62 & 0.69 & 0.32 \\
\hline$\delta_{\text {tol }}$ & $10^{-1}$ & $10^{-2}$ & $10^{-3}$ & $10^{-4}$ & $10^{-5}$ & $10^{-6}$ & $10^{-7}$ & $10^{-18}$ \\
$\epsilon(\%)$ & 59.64 & 14.84 & 19.71 & 4.99 & 1.02 & 0.71 & 0.70 & 0.69 \\
\hline
\end{tabular}

\section{Conclusions}

The new constitutive relation error estimators family introduced has strong mechanical basis and allows us to take into account general (visco)plastic behavior with softening. (Visco)plasticity without softening, elasticity with softening or pure elasticity are particular cases. The dissipation error is also recovered by enforcing a priori the state laws. Extensions to non standard material can be easily done using the concept of bipotential 
introduced by de Saxcé [39]. The implementation of the error in the framework of finite element computation was detailed and the numerical experiments demonstrate that the error takes into account all the numerical parameters of the computation (mesh, time step and stopping criteria). In the future, we plan to apply this error to more complex structures and to introduce specific time, space and iteration indicators for this new family of error estimator; for instance by using the systematic approach introduced in [30].

\section{Acknowledgements}

The second author gratefully acknowledges the support of this work by the U.S. Office of Naval Research under Grant N00014-95-1-0401 and the National Science Foundation under grant ECS-9422707.

\section{A. Duality, convexity and Legendre-Fenchel transform}

Let $A$ and $B$ be two spaces associated through the duality paring:

$$
\begin{aligned}
& a, b \rightarrow a \circ b \\
& A \times B \rightarrow \mathbb{R}
\end{aligned}
$$

The meaning of the ${ }^{\circ}$ operator do not need to be specified. Let a relation between $a \in A$ and $b \in B$ be defined by

$$
b \in \partial_{a} f(a)
$$

where $f(a)$ be a real convex function of a. We recall that a real function $f(a)$ is convex if

$$
f\left(\lambda a_{1}+(1-\lambda) a_{2}\right) \leqslant \lambda f\left(a_{1}\right)+(1-\lambda) f\left(a_{2}\right) \quad \forall \lambda \in(0,1), \quad \forall a_{1}, a_{2} \in A
$$

and strictly convex if the above inequality is satisfied in a strict sense, $\forall a_{1}, a_{2} \in A, a_{1} \neq a_{2}$.

Defining $f^{*}(b)$ as the Legendre-Fenchel transform of $f(a)$ :

$$
f^{*}(b)=\sup _{a^{\prime} \in A}\left(a^{\prime} \circ b-f\left(a^{\prime}\right)\right)
$$

we may write (A.1) in the equivalent dual form

$$
a \in \partial_{b} f^{*}(b)
$$

where $f^{*}(b)$ is convex (property of the Legendre-Fenchel transform). A third equivalent form to (A.1) and (A.4) is

$$
f(a)+f^{*}(b)-a \circ b=0
$$

Moreover, one can show that

$$
f(a)+f^{*}(b)-a \circ b \geqslant 0, \quad \forall(a, b) \in A \times B
$$

\section{References}

[1] P. Ladevèze, Les bases de la méthode de l'erreur en relation de comportement pour le contrôle des calculs éléments finis: Les travaux des années 1975. Rapport interne 163, LMT, Ecole Normale Supérieure de Cachan, 1995.

[2] P. Ladevèze and D. Leguillon, Error estimate procedure in the finite element method and application, SIAM J. Numer. Anal. 20(3) (1983) 485-509.

[3] P. Ladevèze, J.P. Pelle and P. Rougeot, Error estimation and mesh optimization for classical finite elements. Engrg. Comput. 8 (1991) 69-80.

[4] I. Babuska and W.C. Rheinboldt, A posteriori estimates for the finite element method, Int. J. Numer. Methods Engrg. 12 (1978) 1597-1615.

[5] I. Babuska and W.C. Rheinboldt, Adaptative approaches and reliability estimators in finite element analysis, Comput. Methods Appl. Mech. Engrg. 17/18 (1979) 519-540.

[6] D.W. Kelly, J. Gago, O.C. Zienkiewicz and I. Babuska, A posteriori error analysis and adaptative processes in finite element method. Part 1: Error analysis, Int. J. Numer. Methods Engrg. 19 (1983) 1593-1619. 
[7] J. Gago, D.W. Kelly, O.C. Zienkiewicz and I. Babuska, A posteriori errer analysis and adaptative processes in finite element method. Part 2: Adaptative mesh refinement, Int. J. Numer. Methods Engrg. 19 (1983) 1921-1656.

[8] J.T. Oden, L. Demkowicz, W. Rachowicz and T.A. Westermann, Toward a universal $h-p$ adaptative finite element strategy, Part 2: A posteriori error estimation, Comput. Methods Appl. Mech. Engrg. 77 (1989) 113-180.

[9] M. Ainsworth and J.T. Oden, A posteriori error estimation in finite element analysis, Comput. Methods Appl. Mech. Engrg. 142(1-2) (1997) 1-88.

[10] O.C. Zienkiewicz and J.Z. Zhu, A simple error estimator and adaptative procedure for practical engineering analysis, Int. J. Numer. Methods Engrg. 24 (1987) 337-357.

[11] O.C. Zienkiewicz and J.Z. Zhu, The superconvergent patch recovery and a posteriori error estimates, Part 1: The recovery technique, Int. J. Numer. Methods Engrg. 33 (1992) 1331-1364.

[12] O.C. Zienkiewicz and J.Z. Zhu, The superconvergent patch recovery and a posteriori error estimates, Part 2: Error estimates and adaptativity, Int. J. Numer. Methds Engrg. 33 (1992) 1365-1382.

[13] I. Babuska, T. Strouboulis, C.S. Upadhyay, S.K. Gangaraj and K. Copps, Validation of a posteriori error estimators by numerical approach, Int. J. Numer. Methods. Engrg., 37 (1994) 1073-1123.

[14] I. Babuska, T. Strouboulis and C.S. Upadhyay, A model study of the quality of a posteriori error estimators for linear elliptic problems: Error estimation in the interior of patchwise uniform grids of triangles, Comput. Methods Appl. Mech. Engrg. 114 (1994) $307-378$.

[15] I. Babuska and W.C. Rheinboldt, Computational error estimates and adaptative processes for some nonlinear structural problems, Comput. Methods Appl. Mech. Engrg. 34 (1982) 895-937.

[16] C. Johnson and P. Hansbo, Adaptative finite element methods in computational mechanics, Comput. Methods Appl. Mech. Engrg. 101 (1992) 143-181.

[17] R. Verfiirth, A Review of A Posteriori Error Estimation and Adaptive Mesh-Refinement Techniques, Wiley-Teubner, ISBN 0-471-96795-5, 1996.

[18] J.M. Bass and J.T. Oden, Adaptative finite element methods for a class of evolution problems in viscoplasticity, Int.J. Engrg. Sci. 25(6) (1987) 623-653.

[19] D. Aubry and B. Tie, Relationships between error estimation and adaptive computations in strain localization, in: Proc. 9th Conf. on Engineering Mechanics, College Station, 1992.

[20] D. Peric, J. Yu and D.R.J. Owen, On error estimates and adaptativity in elasto-plastic solids: Applications to the numerical simulation of strain localisation in classical and Cosserat continua, Int. J. Numer. Methods Engrg. 37(8) (1994) 1351-1379.

[21] G. Pijaudier-Cabot, L. Bode and A. Huerta, Arbitrary Lagrangian-Eulerian finite element analysis of strain localization in transient problems, Int. J. Numer. Methods Engrg. 38(24) (1995) 4171-4191.

[22] P. Bussy, Optimisation et fiabilité des calculs par éléments finis en non linéarité géométrique. Thesis, Université Paris 6, 1984.

[23] P. Bussy, G. Coffignal and P. Ladevèze. Reliability and optimization of non linear finite element analyses, in: Proc. of NUMETA'85, Swansea, 1985.

[24] O.C. Zienkiewicz and G.C. Huang, Note on localization phenomena and adaptive finite-element analysis in forming processes, Comm. Appl. Numer. Methods 6(2) (1990) 71-76.

[25] L. Fourment and J.L. Chenot, Error estimators for viscoplastic materials: application to forming processes, Int. J. Numer. Methods Engrg. 12(5) (1995) 469-490.

[26] D.C. Drucker, On the postulate of stability of material in the mechanics of continua. Journal de Mécanique 3(2) (1964) $235-249$.

[27] P. Ladevèze, Sur une famille d'algorithmes en mécanique des structures, Compte-Rendus Acad. Sci. Paris, Série II 300 (1985) $41-44$.

[28] P. Ladevèze, G. Coffignal and J.P. Pelle, Accuracy of Elastoplastic and Dynamic Analysis, in: I. Babuska, J. Gago, E. Oliveira and O.C. Zienkiewicz, eds., Accuracy Estimates and Adaptative Refinements in Finite Element Computations (J. Wiley, 1986) 181-203.

[29] L. Gallimard, P. Ladevèze and J.P. Pelle, Error estimation and adaptativity in elastoplasticity, Int. J. Numer. Methods Engrg. 39 (1996) 189-217.

[30] P. Ladevèze and N. Moës, A posteriori constitutive relation error estimators for nonlinear finite element analysis and adaptive control, in: P. Ladevèze and J.T. Oden, eds., Advances in Adaptive Computational Methods (Elsevier Publication, 1997) 231-256.

[31] P. Ladevèze, La méthode à grand incrément de temps pour l'analyse de structures à comportement non linéaires décrit par variables internes, Compte-Rendus Acad. Sci. Paris, Série II 309(11) (1989) 1095-1099.

[32] P. Ladevèze, Mécanique non linéaire des structures. Nouvelle approche et méthodes de calcul non incrémentales. Hermes, 1996. (English version, Springer, 1998).

[33] P. Ladevèze and N. Møës, A new a posteriori error estimation for nonlinear time-dependent finite element analysis, Comput. Methods Appl. Mech. Engrg. 157 (1998) 45-68.

[34] P. Ladevèze and N. Moës, Adaptive control for finite element analysis in plasticity, Comput. Struct. To appear in a special issue Advances in Engineering Software Incorporating Computing Systems in Engineering.

[35] P. Ladevèze and E. Le Dantec, Damage modelling of the elementary ply for the laminated composites, Comp. Sci. Technol. 43 (1992) 257-267.

[36] P. Ladevèze, A damage computational method for composite structures, Comput. Struct. 44 (1992) 79-87.

[37] P. Ladevèze, Nouvelles procédures d'estimations d'erreur relative à la méthode des éléments finis, in: Proc. Joumées Eléments finis, Rennes, 1977.

[38] P. Coorevits, P. Ladevèze, J.P. Pelle and P. Rougeot, Some new applications of a method for the control and optimization of finite element computations. In P. Ladevèze and O.C. Zienkiewicz, eds., New Advances in Computational Structural Mechanics (Elsevier, 1992) 205-217.

[39] G. de Saxcé, A generalization of Fenchel's inequality and its applications to constitutive law, Compte-Rendus Acad. Sci. Paris, Série II 314 (1992) 125-129. 